\title{
A rare case of unruptured tubal pregnancy at ten weeks of gestation
}

Aryal S', Thapa $\mathbf{M}^{2}$, Karki $\mathbf{C}^{3}$

${ }^{1}$ Dr. Shreyashi Aryal, Resident; ${ }^{2}$ Dr. Meena Thapa, Associate Professor; ${ }^{3}$ Dr. Chanda Karki, Professor, Department of Obstetrics and Gynaecology, Kathmandu Medical College Teaching Hospital, Kathmandu, Nepal.

\section{Abstract}

Ectopic pregnancy is a life threatening condition and can present itself in diverse ways. This case reports a 23 years primigravida, admitted with history of amenorrhoea of ten weeks and right sided unruptured tubal pregnancy diagnosed on routine ultrasonography. On laparoscopy, there was an unruptured tubal pregnancy with gestational sac measuring around $6 \times 5 \mathrm{~cm}$. The foetus had crown rump length of $32 \mathrm{~mm}$ and foot length of $5 \mathrm{~mm}$; both of which correspond probably to 10 to 11 weeks size. Segmental resection of the affected part of the fallopian tube was done laparoscopically. Rarely tubal pregnancy can remain asymptomatic and unruptured for longer duration of gestation than the usual scenario. The reported average duration of diagnosis of unruptured tubal pregnancy is of nine weeks and this case explores the possibility of removing an intact foetus at longer gestational age through laparoscopy.

Key words: ectopic pregnancy, laparoscopy, tubal pregnancy

\section{INTRODUCTION}

$\mathrm{E}^{\mathrm{c}}$ ctopic pregnancy is a life threatening condition but it presents in various ways and is not always symptomatic. The incidence of ruptured ectopic pregnancy presenting in a critical stage has significantly decreased because of combined diagnostic modality of transvaginal sonography and measurement of serum beta-human chorionic gonadotropin (hCG). Nevertheless, ectopic pregnancies remain an important cause of morbidity and mortality because the modern diagnostic tools and therapeutic facilities are not always available in most institutions in the country.

\section{CASE REPORT}

A 23 years primigravida, attended the Obstetrics and Gynaecology outpatient department of Kathmandu Medical College, for her regular antenatal check up with

Address for correspondence
Dr. Shreyashi Aryal
Resident
Department of Obstetrics and Gynaecology, Kathmandu
Medical College.
Email:shreyashi_aryal@ hotmail.com

history of amenorrhoea of ten weeks, slight dizziness, and one episode of syncope on the previous day.

There was no history of abdominal pain or vaginal bleeding; neither had she any history of high risk conditions related to ectopic pregnancy, like history of subfertility, smoking, use of intra uterine contraceptive device, pelvic inflammatory disease, previous pelvic surgery, or previous ectopic pregnancy etc.

On general examination, she was haemodynamically stable; there was no pallor, tachycardia or fever. There was no rebound tenderness or guarding on abdominal examination. Pelvic examination revealed uterus to be of six weeks size, retroverted, and there was a significant fullness in the right fornix. Cervical motion tenderness could not be elicited.

Her routine antenatal investigations were within normal limits with a haemoglobin level of $12.9 \mathrm{~g} \%$. With the suspicion of ectopic pregnancy, an ultrasonography was advised which was suggestive of a tubal pregnancy. Abdominal ultrasound showed a gestational sac of seven weeks five days in the right adnexal region with a foetus showing normal cardiac activity. A transvaginal 


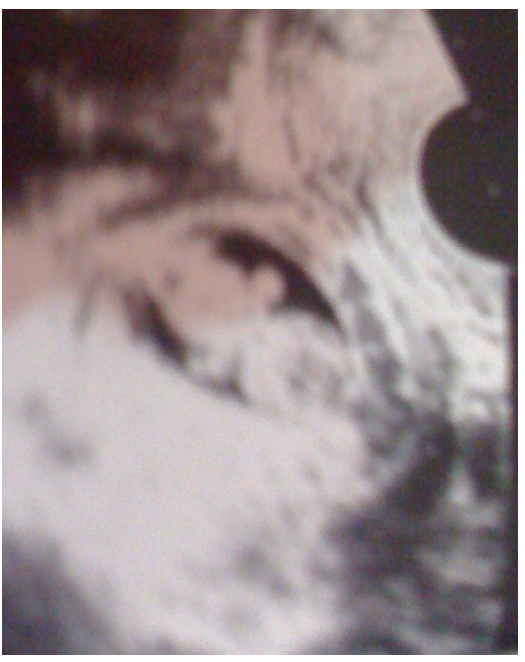

Figure 1: Transvaginal sonogram showing foetus of nine weeks size in the right adnexa

ultrasound was again done to reconfirm the diagnosis and it showed a foetus of nine weeks and one day, with normal cardiac activity, situated in the right adnexa (Figure 1).

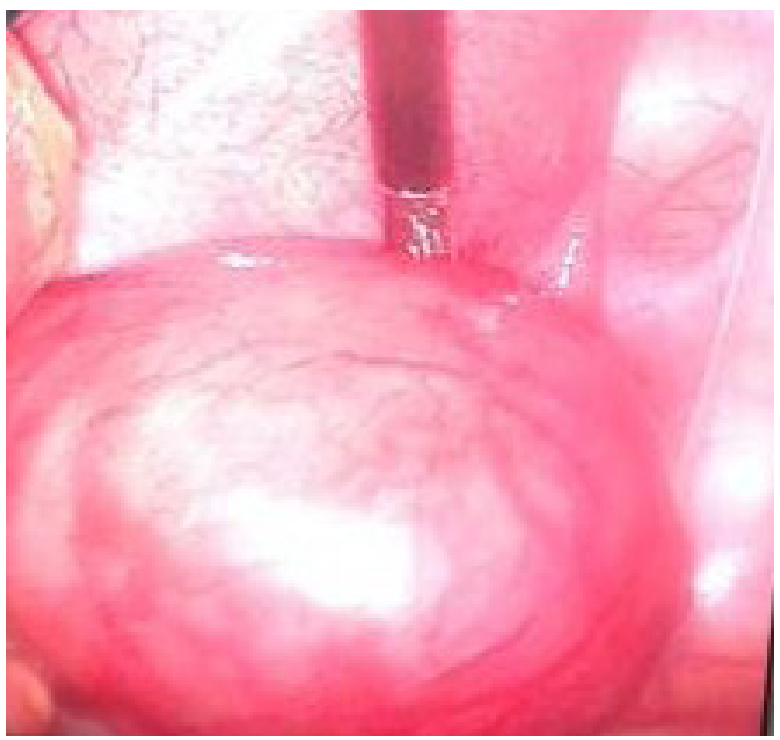

Figure 2: Laparoscopic view of the gestational sac

Since the patient was haemodynamically stable, laparoscopy was done on the second day of admission. There was an unruptured significantly large right tubal swelling in the isthmic region containing gestational sac, with foetus and placenta (Figure 2). The swollen part of the tube was noticeably thick and muscular and the gestational sac measured around $6 \times 5 \mathrm{~cm}$ (Figure 3). Segmental resection of the affected part of the fallopian tube was done and the gestational sac was removed along with it. The gestational sac contained a foetus

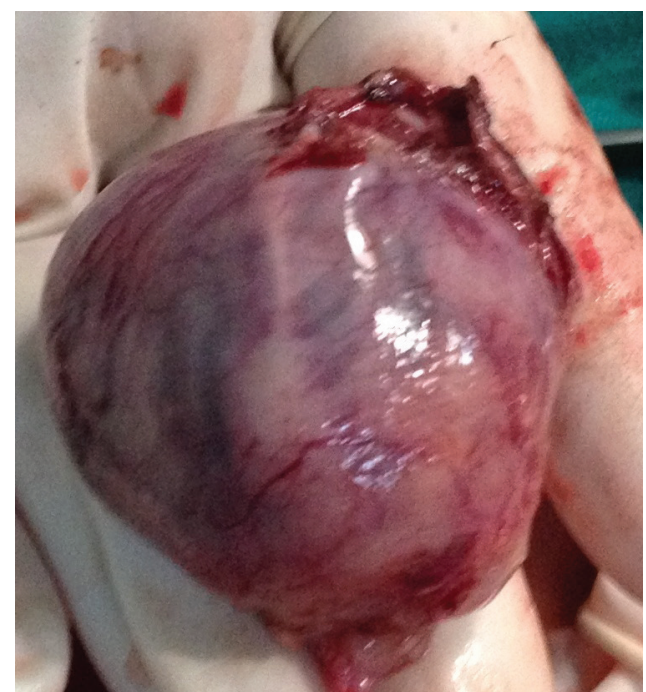

Figure 3: Foetus along with the placenta

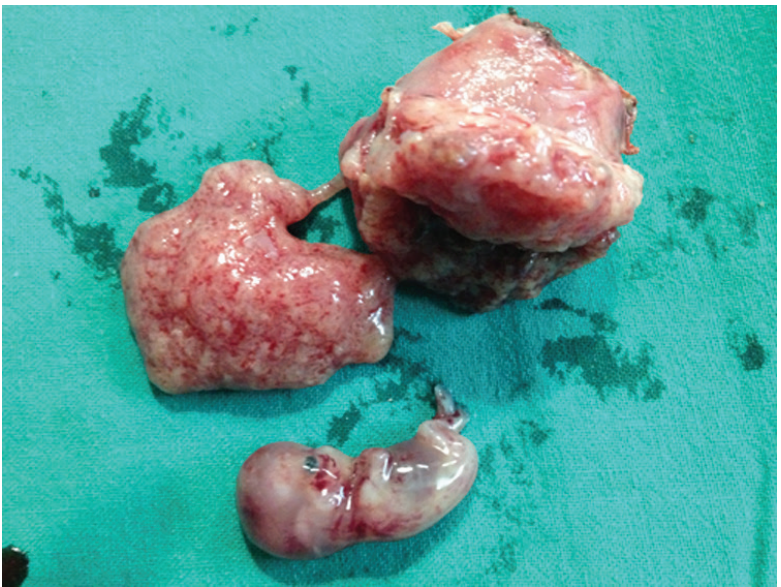

Figure 4: Foetus along with the placenta

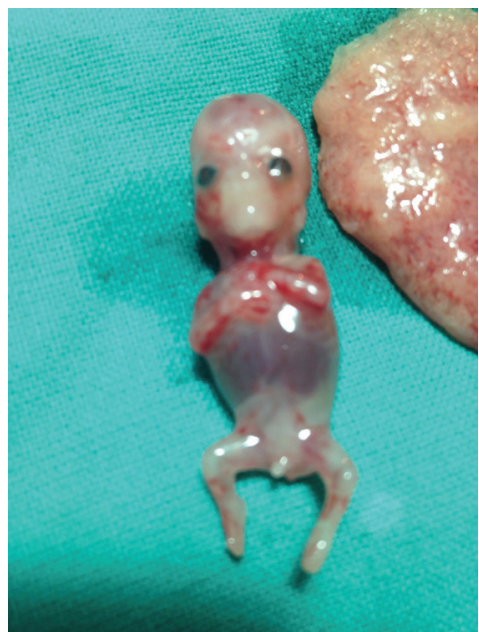

Figure 5: Intact fetus of 10 to 11 weeks size

which had crown rump length of $32 \mathrm{~mm}$. and foot length of $5 \mathrm{~mm}$. both of which correspond to the gestational age of 10 to 11 weeks size ${ }^{1,2}$ (Figure 4). It had well developed 
eyes and limbs with fingernails on gross examination which suggests a gestational age of about eight to ten weeks. Placenta was also of good size weighing $55 \mathrm{~g}$ (Figure 5). Uterus was six weeks size, left tube and both the ovaries were normal.

Postoperative period was uneventful and she was discharged on third postoperative day. Patient had come for follow up after one week and had no significant complaint.

\section{DISCUSSION}

The incidence of ectopic pregnancies shown in various studies in Nepal is 0.68 to $1.8 \%{ }^{3-5}$. The incidence is on the rise probably due to increase in sexually transmitted disease and availability of assisted reproductive techniques in the country. Out of total ectopic pregnancies, $95 \%$ are tubal $[80 \%$ ampullary, $12 \%$ isthmic, $2.4 \%$ interstitial], and ovarian and abdominal pregnancies account for $3.2 \%$ and $1.3 \%$ respectively ${ }^{6}$.

Ectopic pregnancies are easily diagnosed clinically in a ruptured state but the diagnosis in an unruptured state is difficult because there may be neither symptoms nor signs which can be elicited. So the diagnosis is usually made incidentally on ultrasonography but the findings may be sometimes deceptive in diagnosing and assessing the gestational age. Transvaginal ultrasonography has higher sensitivity (98.3\%) and specificity (99.9\%) than abdominal ultrasound for diagnosing ectopic pregnancies ${ }^{7}$. This is probably the reason for the gestational age difference of nine days in transvaginal ultrasonography and transabdominal scan in this case.

The presence of an adnexal gestational sac with a foetal pole and cardiac activity is seen is the most specific sign of ectopic pregnancy, but such sign is seen in only $10 \%$ to $17 \%$ of cases $^{8}$.

Ectopic pregnancies usually present at around six to eight weeks gestation and the most common site is the ampullary region of the fallopian tube. It is usually difficult for a pregnancy to continue in an ectopic site without rupture especially for longer than this duration and it is more so in the isthmic region'. After eight weeks of gestation, the usual presentation is in a ruptured state and silent presentation like in this case is remarkable.
In ectopic pregnancies, future fertility is a serious concern and in this case there is good fertility outcome since the left tube and ovary were normal ${ }^{3}$.

Most studies show average duration of eight weeks when unruptured ectopic pregnancy was diagnosed. Longer duration was shown in a few studies ${ }^{10}$. Ju R and Perretta in 2011 reported a case of tubal pregnancy consistent with 10.1 weeks gestation calculated by a crown rump length of $3.4 \mathrm{~cm}$ which was removed by laparoscopic right salpingectomy ${ }^{11}$. Another case of tubal pregnancy reported in 2008 by Yeung and Pasic at nine+ weeks, also managed with laparoscopic salpingectomy ${ }^{12}$.

Laparoscopy has become the recommended approach of treatment of ectopic pregnancies in most cases. Laparotomy is usually reserved for patients who are haemodynamically unstable or patients with cornual ectopic pregnancies. Laparoscopy has various advantages like fewer postoperative adhesions, significantly less blood loss, reduced need for analgesia, reduced cost, hospitalization, and convalescence period to name a few.

Rarely tubal pregnancy can remain asymptomatic and unruptured for longer duration of gestation than the usual scenario. The reported average duration at diagnosis of unruptured tubal pregnancy is of nine weeks and this case explores the possibility of removing an intact foetus at longer gestational age through laparoscopy. Laparoscopic management consists of either conservative surgeries like linear salpingostomy or segmental resection or a more aggressive treatment with salpingectomy. Segmental resection with either immediate or delayed anastomosis appears preferable to linear salpingostomy for the conservative management of unruptured isthmic ectopic pregnancy as there is a higher tubal patency rate and normal intrauterine pregnancy ${ }^{13}$.

\section{CONCLUSION}

Unruptured ectopic pregnancy, can rarely present at late gestational age without any symptoms. Timely diagnosis and treatment with minimal invasive surgery helps to reduce maternal morbidity and mortality. 


\section{REFERENCES}

1. Loughna P, Chitty L, Evans T, Chudleigh T. Fetal size and dating: charts recommended for clinical obstetric practice. Ultrasound. 2009 Aug;17(3).

2. Hern W. Correlation of Fetal Age and Measurements between 10 and 26 Weeks of Gestation. Obstet Gynecol. 1984 Jan;63(1):26-32.

3. Karki C, Karki A, Yangzom K. Ectopic pregnancy and its effect on future fertility. J South Asian Fed Obstet Gynaecol. 2009 Jan-Apr;1(1):35-9.

4. Gurung G, Rana A. Diagnostic dilemma in cases of ectopic pregnancy: a five year prospective study at Tribhuvan University Teaching Hospital. J Inst Med. 2006;28(1).

5. Karki L, Pradhan B, Duwa S. Annual analysis of ectopic pregnancy in tertiary care hospital: Postgrad Med J. 2011 Jan-Jun;11(1):5-8.

6. Bouyer J, Coste J, Fernandez H. Sites of ectopic pregnancy: a 10 year population cased study of 1800 cases. Hum Reprod. 2002;17(12):3224-30.

7. Kirk E, Bourne T. Diagnosis of ectopic pregnancy with ultrasound. Best Pract Res Clin Obstet Gynaecol. 2009:1-8.

8. Winder S, Reid S,Condous G. Ultrasound diagnosis of ectopic pregnancy. AJUM. 2011 May;14(2):2933.

9. Senterman M, Jibodh R, Tulandi T. Histopathologic study of ampullary and isthmic tubal ectopic pregnancy. Am J Obstet Gynecol. 1988 Oct;159(4):939-41.

10. Obed SA. Diagnosis of unruptured ectopic pregnancy is still uncommon in Ghana. Ghana Med J. 2006 March;40(1):3-7.

11. Ju R, Perretta T, Chang PL. Large intact tubal ectopic pregnancy removed via laparoscopy. The Female Patient. 2011;36(6):40-2.

12. Yeung PP, Pasic RP. Intact ectopic pregnancy found at laparoscopic salpingectomy. J Minim Invasive Gynecol. 2008;15(4):393.

13. DeCherney AH, Boyers SP. Isthmic ectopic pregnancy: segmental resection as the treatment of choice.Fertil Steril.1985 Sep;44(3):307-12. 